\title{
Comparison of qSOFA score, SOFA score, and SIRS criteria for the prediction of infection and mortality among surgical intermediate and intensive care patients
}

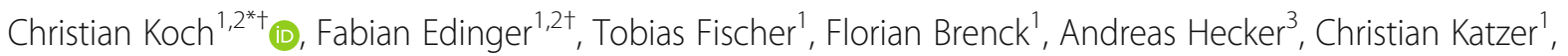
Melanie Markmann ${ }^{1}$, Michael Sander ${ }^{1,2}$ and Emmanuel Schneck ${ }^{1,2}$

\begin{abstract}
Background: It is crucial to rapidly identify sepsis so that adequate treatment may be initiated. Accordingly, the Sequential Organ Failure Assessment (SOFA) and the quick SOFA (qSOFA) scores are used to evaluate intensive care unit (ICU) and non-ICU patients, respectively. As demand for ICU beds rises, the intermediate care unit (IMCU) carries greater importance as a bridge between the ICU and the regular ward. This study aimed to examine the ability of SOFA and qSOFA scores to predict suspected infection and mortality in IMCU patients.

Methods: Retrospective data analysis included 13,780 surgical patients treated at the IMCU, ICU, or both between January 01, 2012, and September 30, 2018. Patients were screened for suspected infection (i.e., the commencement of broad-spectrum antibiotics) and then evaluated for the SOFA score, qSOFA score, and the 1992 defined systemic inflammatory response syndrome (SIRS) criteria.

Results: Suspected infection was detected in 1306 (18.3\%) of IMCU, 1365 (35.5\%) of ICU, and 1734 (62.0\%) of IMCU/ ICU encounters. Overall, 458 (3.3\%) patients died (IMCU 45 [0.6\%]; ICU 250 [6.5\%]; IMCU/ICU 163 [5.8\%]). All investigated scores failed to predict suspected infection independently of the analyzed subgroup. Regarding mortality prediction, the QSOFA score performed sufficiently within the IMCU cohort (AUCROC SIRS 0.72 [0.71-0.72]; SOFA 0.52 [0.51-0.53]; qSOFA 0.82 [0.79-0.84]), while the SOFA score was predictive in patients of the IMCU/ICU cohort (AUCROC SIRS 0.54 [0.53-0.54]; SOFA 0.73 [0.70-0.77]; qSOFA 0.59 [0.58-0.59]).

Conclusions: None of the assessed scores was sufficiently able to predict suspected infection in surgical ICU or IMCU patients. While the qSOFA score is appropriate for mortality prediction in IMCU patients, SOFA score prediction quality is increased in critically ill patients.
\end{abstract}

Keywords: Sepsis, Critical care, qSOFA, SOFA, Mortality, Infections

\footnotetext{
* Correspondence: Christian.koch@chiru.med.uni-giessen.de

${ }^{+}$Christian Koch and Fabian Edinger contributed equally to this work.

'Department of Anesthesiology, Operative Intensive Care Medicine and Pain

Therapy, University Hospital of Giessen, Rudolf-Buchheim-Street 7, 35392

Giessen, Germany

${ }^{2}$ German Center of Infection Research (DZIF), Partner Site Giessen/Marburg/

Langen, Giessen, Germany

Full list of author information is available at the end of the article
}

(c) The Author(s). 2020 Open Access This article is licensed under a Creative Commons Attribution 4.0 International License, which permits use, sharing, adaptation, distribution and reproduction in any medium or format, as long as you give appropriate credit to the original author(s) and the source, provide a link to the Creative Commons licence, and indicate if changes were made. The images or other third party material in this article are included in the article's Creative Commons licence, unless indicated otherwise in a credit line to the material. If material is not included in the article's Creative Commons licence and your intended use is not permitted by statutory regulation or exceeds the permitted use, you will need to obtain permission directly from the copyright holder. To view a copy of this licence, visit http://creativecommons.org/licenses/by/4.0/ The Creative Commons Public Domain Dedication waiver (http://creativecommons.org/publicdomain/zero/1.0/) applies to the data made available in this article, unless otherwise stated in a credit line to the data. 


\section{Background}

Sepsis is defined as a life-threatening disease complex characterized by severe organ dysfunction resulting from a dysbalanced host response to an infection [1]. Despite modern treatment protocols, sepsis-related mortality remains highly associated with delays in adequate treatment [2]. For this reason, modern clinical concepts have focused on the development of criteria aiming for the rapid identification of sepsis [3, 4].

For 24 years, sepsis has been defined as suspected or proven infection, together with two or more systemic inflammatory response syndrome (SIRS) criteria [5]. However, during the last decade, clinical characteristics that serve to define sepsis changed due to an improved understanding of the underlying pathobiology. Therefore, in 2016, the Third International Consensus Definitions for Sepsis and Septic Shock (Sepsis-3) introduced a significant change in the approach to the definition and diagnostic criteria of sepsis [1].

Nevertheless, a highly sensitive and specific diagnostic test for the detection of sepsis is currently still lacking. Among ICU encounters with suspected infection, the Sepsis-3 Task Force recommended the use of the Sequential (sepsis-related) Organ Failure Assessment (SOFA) score for the identification of septic patients $[1,6]$. For the rapid identification of patients with suspected infection outside of the ICU, on the other hand, Seymour et al. introduced the quick Sequential Organ Failure Assessment (qSOFA) score [7]. The qSOFA score is a simple score consisting of three items: respiratory rate $(\mathrm{RR}) \geq 22$ breaths per minute, altered mentation (Glasgow Coma Scale $[\mathrm{GCS}]<15$ ), and systolic blood pressure $(\mathrm{SBP})<$ $100 \mathrm{mmHg}$. A qSOFA score $\geq 2$ was found to be significantly predictive of increased all-cause mortality in patients outside of the ICU [7]. Therefore, the authors of the Third International Consensus Definitions for Sepsis and Septic Shock (Sepsis-3) recommended the use of the qSOFA score for the identification of adult septic patients in out-of-hospital, emergency department, or general hospital ward settings [1].

Intermediate care units (IMCUs) are logistically situated between ICUs and general wards and serve as an alternative care setting for patients deemed too unstable to be cared for on the general ward, but without requiring the resources of an ICU [8-10]. Lacking a unitary definition of IMCUs, their nomenclature varies from high dependency, progressive care, medium care, or step-down units, resulting in a high variability of organizational practice [8]. While most IMCUs offer continuous monitoring of vital signs, the ability to provide mechanical ventilation, renal replacement therapy, and differentiated catecholamine therapy is normally limited [11]. Although IMCU patients commonly demand a higher level of nursing compared to the normal ward, the severity of illness is lower than on the ICU $[12,13]$. It is worth noting that the mere presence of an IMCU is associated with a significantly reduced hospital mortality in ICU patients, underlining the impact of an IMCU as a bridge between the ICU and the regular ward [14]. Furthermore, by demonstrating that septic shock patients can be successfully treated on an IMCU, Meaudre et al. proposed the potential of this critical care resource [15]. Surgical patients, in particular, are often treated in IMCUs because they are commonly extubated shortly after surgery and are therefore not mandatorily eligible for ICU treatment. However, surgical patients are also at risk for postoperative infections. Clinical signs of infection in these patients are challenging, since they might also be caused by the surgery itself, implicating the need for thorough risk stratification [16, 17]. Lacking evidence, it is not yet defined whether these patients should be evaluated as ICU or non-ICU patients when it comes to the identification of sepsis, respectively severe infection, raising the question as to whether the SOFA or qSOFA score should be used. For this reason, there are currently no specific recommendations for the screening of septic patients treated on IMCU. Therefore, the aim of our study was to compare the predictive power of qSOFA and SOFA scores, as well as the 1992 defined SIRS criteria, for mortality or infection in a large sample of surgical ICU and IMCU patients. We hypothesized that the qSOFA score would perform superiorly to the SOFA score and SIRS criteria in predicting mortality or infection among IMCU patients.

\section{Methods}

\section{Study design and patient recruitment}

This retrospective, 6-year cohort study was approved by the local ethics committee (Justus-Liebig-University, Giessen, Germany, trial code 240/16). The methods and results are presented in accordance with the Strengthening the Reporting of Observational Studies in Epidemiology (STROBE) guidelines. Data of all patients aged $\geq 18$ years with suspected infection who were treated at the surgical ICU and/or IMCU of the University Hospital of Giessen between January 01, 2012, and September 30, 2018, were included.

\section{Data acquisition}

After identification of patients, study data were automatically extracted from the local patient data management system (ICU-Data ${ }^{\circ}, \mathrm{IMESO}^{\circ} \mathrm{GmbH}$, Giessen, Germany) with Structured Query Language and Procedural Language (SQL/PL-SQL)-based scripts. Patients' characteristics included age, body mass index (BMI), treatment unit (ICU, IMCU, or both), Acute Physiology and Chronic Health Evaluation (APACHE) II score, and, if applicable, the type of performed surgery. Episodes of suspected 
infections were defined as the first $72 \mathrm{~h}$ after starting treatment with broad-spectrum antibiotic agents, which included carbapenems, glycopeptides, quinolones, piperacillin/sulbactam, ceftazidime, cefepime, linezolid, tigecycline, daptomycin, and fosfomycin. Contrarily, the following antibiotics were excluded because they did not meet the definition of broad-spectrum antibiotic treatment, according to the European and local sepsis guidelines [18]: ampicillin, cefazolin, cefuroxime, colistin, metronidazole, erythromycin, trimethoprim/sulfamethoxazole, and azithromycin.

While the SOFA score was recorded daily throughout the patient's ICU treatment by the attending physician, SIRS criteria and qSOFA score were not registered systematically and therefore needed to be calculated retrospectively. First, relevant vital signs (respiratory rate, systolic blood pressure, heart rate, temperature), which were automatically recorded every $15 \mathrm{~min}$, were systematically analyzed for outliers. For this purpose, a second data table was built, and the median for each parameter was calculated. For the calculation of the median of the respiratory and heart rates as well as the systolic blood pressure, three values of each time point were included (i.e., corresponding time point and two values aside). Since extreme values of both parameters were possible in critically ill patients, no absolute thresholds were defined as outliers. Secondly, the median for each temperature time point was calculated out of seventeen values (i.e., corresponding time point and sixteen values aside) to equalize incorrect measurements, which can be caused by a dislocated temperature probe. Body temperature measurements $\leq 31{ }^{\circ} \mathrm{C}$ were defined as artefacts and therefore excluded. If GCS was not available, Richmond Agitation Sedation Scale (RASS) was used for the assessment of consciousness (where RASS $\neq 0$ was defined as the fulfillment of "altered mental status," respectively as GCS $\leq 15$ ). Leucocyte count was derived from the daily routine blood cell count, while arterial carbon dioxide partial pressure $\left(\mathrm{paCO}_{2}\right)$ was extracted from the blood gas analyses, which was most recent to the analyzed time frame.

In accordance with their definitions, the SIRS criteria and the qSOFA score were rated positive if at least two criteria were fulfilled during a minimum of $30 \mathrm{~min}[7,19]$. The SOFA score of each day was compared with the value of the previous day. An increase of at least two points was rated positive.

Furthermore, the outcome analysis included the need for and duration of invasive ventilation, requirement for catecholamines, length of hospital stay, and hospital mortality.

\section{Statistical analysis}

All encounters were divided into three subgroups, according to their location of treatment (IMCU only, ICU only, or both [IMCU/ICU]). In cases of normal distribution of the data, the results are expressed as mean \pm standard deviation (SD) and, in cases where data were not normally distributed, as median (interquartile range $[\mathrm{IQR}]$ ). Receiver operating characteristic curves (ROC) were used for calculation of the predictive validity of the SIRS criteria, qSOFA score, and SOFA score. The primary aims of these analyses were defined as the identification of suspected infection and the prediction of mortality, assessed by means of the area under the ROC curve (AUCROC). Furthermore, sensitivity and specificity of both primary aims were calculated. AUCROCs were considered to be poor at $0.51-0.69$, adequate at $0.7-0.79$, sufficient at $0.8-0.89$, and excellent at 0.9 or higher. AUCROCs are displayed with the 95\% confidence interval. Data were tested for statistically significant differences using chi-squared test or Fisher's exact test, when appropriate. A two-tailed value of $p<0.05$ was considered to be statistically significant. All statistical analyses were performed using the R statistical software version 3.5.1 (www.r-project.org).

\section{Results}

\section{Characteristics of the study cohorts}

For the observational period, 13,780 patients were identified. Of these, $7133(51.8 \%)$ were treated only at the IMCU, $3850(27.9 \%)$ at the ICU, and $2797(20.3 \%)$ at both the ICU and IMCU (Fig. 1). Patients' characteristics, underlying departments, and outcome parameters are shown in Table 1. Overall, 458 (3.3\%) subjects died within the observation period (IMCU 45 [0.6\%]; ICU 250 [6.5\%]; IMCU/ICU 163 [5.8\%]). Suspected infections were identified in 4405 (32.0\%) patient encounters (IMCU 1306 [18.3\%]; ICU 1365 [35.5\%]; ICU/IMCU 1734 [62.0\%]; Fig. 1).

\section{Performance of clinical scores in the IMCU}

Among 1306 IMCU patients with suspected infection, 1023 (78.3\%) fulfilled at least two positive SIRS criteria. Furthermore, a SOFA score increase was detected in 65 (5.0\%) cases, while qSOFA scoring was positive in 735 (56.3\%) patients.

Overall, the predictive performance of the scores of interest was low. However, compared to the SOFA score, the SIRS criteria and qSOFA score performed superiorly regarding their discrimination between suspected infection and the use of broad-spectrum antibiotics (SIRS: AUCROC $=0.63[0.62-0.65] ;$ SOFA: AUCROC $=0.52$ [0.51-0.53]; qSOFA: AUCROC $=0.63$ [0.62-0.65]; SIRS vs. SOFA: $p<0.001$; qSOFA vs. SOFA: $p<0.001$; SIRS vs. qSOFA: $p=0.833$; Fig. 2 ). While the highest sensitivity for the detection of presumed sepsis was achieved by means of the SIRS criteria, the maximum specificity was found with the SOFA score (Table 2). 


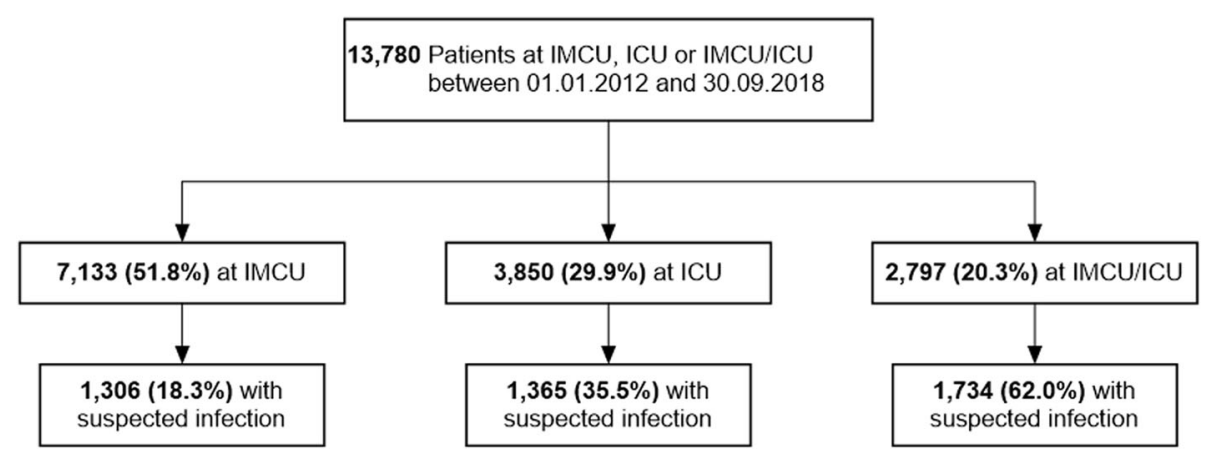

Fig. 1 Composition of the different groups. ICU, intensive care unit; IMCU, intermediate care unit

All IMCU patients with suspected infection who died (45 [3.4\%]) fulfilled at least two SIRS criteria, while the SOFA score was positive in 12 (26.7\%) lethal cases and the qSOFA score in 44 (97.8\%) of those who died. The highest predictive validity for hospital mortality was achieved by calculating the qSOFA score, while SIRS criteria and SOFA score performed significantly inferiorly regarding their predictive validity (SIRS: AUCROC = 0.72 [0.71-0.72]; SOFA: AUCROC $=0.63$ [0.56-0.69]; qSOFA: AUCROC $=0.82$ [0.79-0.84]; SIRS vs. SOFA: $p$ $=0.006$; qSOFA vs. SOFA: $p<0.001$; SIRS vs. qSOFA: $p$ $<0.001$; Fig. 3). SIRS criteria and qSOFA score reached high sensitivity and low specificity regarding mortality, while the SOFA score revealed contrary results (Table 3).

\section{Performance of clinical scores in the ICU}

Of 1635 ICU encounters with suspected infection, a SOFA score increase was identified in 446 (32.7\%) encounters, while qSOFA scoring was positive in 1111 (81.4\%) cases. In 1276 (93.5\%) encounters, at least two SIRS criteria were fulfilled.

Overall, the discriminative power for the identification of patients receiving broad-spectrum antibiotic treatment in the cohort of patients with suspected infection was poor (SIRS: AUCROC $=0.63[0.62-0.64]$; SOFA:
AUCROC $=0.65[0.64-0.66] ;$ qSOFA: AUCROC $=0.66$ [0.65-0.68]; SIRS vs. SOFA: $p=0.008$; qSOFA vs. SOFA: $p=0.098$; SIRS vs. qSOFA: $p<0.001$; Fig. 2). SIRS criteria and qSOFA score were highly sensitive but not specific for presumed sepsis, while contrary results were demonstrated for the SOFA score (Table 2).

Overall, 250 (15.3\%) ICU patients with suspected infection deceased. A majority of these subjects showed positive SIRS criteria (241 [96.4\%]) and qSOFA score (240 [96.0\%]), while positive SOFA was detected only in $122(48.8 \%)$ encounters. SIRS criteria and qSOFA score reached high sensitivity but low specificity regarding the prediction of mortality, while SOFA score revealed contrary results (Table 3). Overall, the predictive validity of all included scores was poor. However, compared to SIRS criteria, SOFA and qSOFA scores performed superiorly regarding the prediction of mortality (SIRS: AUCROC $=0.60[0.59-0.62] ;$ SOFA: AUCROC $=0.69$ [0.66-0.72]; qSOFA: AUCROC $=0.69$ [0.68-0.71]; SIRS vs. SOFA: $p<0.001$; qSOFA vs. SOFA: $p=0.92$; SIRS vs. qSOFA: $p<0.001$; Fig. 3).

\section{Clinical scores in patients treated at the ICU and IMCU}

Among the 1734 (62.0\%) encounters with suspected infection in patients who were admitted to the IMCU and ICU, 1676 (96.7\%) showed at least two positive SIRS

Table 1 Basic patient characteristics

\begin{tabular}{lllll}
\hline Parameter & IMCU & ICU & IMCU/ICU & All \\
\hline Age (years) & $61[41-76]$ & $64[52-75]$ & $69[57-78]$ & $64[49-76]$ \\
BMI $\left(\mathbf{k g} / \mathbf{m}^{2}\right)$ & $26.8[23.9-31.0]$ & $26.2[23.4-29.8]$ & $26.7[23.9-30.9]$ & $26.6[23.7-30.7]$ \\
APACHE II & $4[0-12]$ & $12[0-19]$ & $18[14-23]$ & $10[0-17]$ \\
Invasive ventilation & $1.9 \%$ & $52.3 \%$ & $67.0 \%$ & $29.2 \%$ \\
Need for catecholamines & $4.3 \%$ & $38.0 \%$ & $57.1 \%$ & $24.4 \%$ \\
Hospital stay & $3.12 \pm 11.91$ & $4.80 \pm 12.34$ & $23.11 \pm 33.79$ & $7.65 \pm 20.22$ \\
Hospital mortality & $0.6 \%$ & $6.5 \%$ & $5.8 \%$ & $3.3 \%$ \\
Infection & $18.3 \%$ & $35.5 \%$ & $62.0 \%$ & $32.0 \%$ \\
\hline
\end{tabular}

Data are expressed as median with interquartile range (IQR), percentage, or, if normally distributed, as mean with standard deviation ( \pm ) APACHE II Acute Physiology and Chronic Health Evaluation, BMI Body mass index, ICU intensive care unit, IMCU Intermediate care unit 

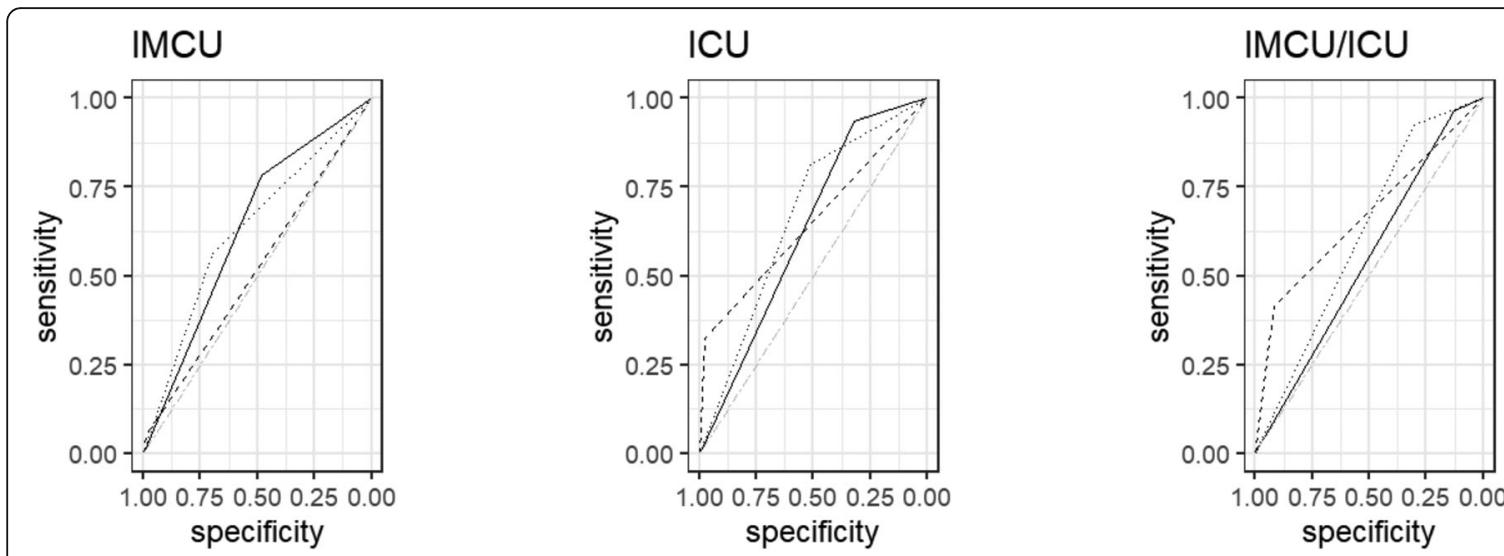

Scores

- SIRS

SOFA

qSofa

Fig. 2 Predictive validity for suspected infection using clinical scores. Receiver operating characteristic curves for positive SIRS criteria, SOFA score, and qSOFA score are pictured. ICU, intensive care unit; IMCU, intermediate care unit; qSOFA, quick Sequential Organ Failure Assessment; SIRS, systemic inflammatory response syndrome; SOFA, Sequential Organ Failure Assessment

criteria, while SOFA and qSOFA scores were positive in $721(41.6 \%)$ and 1607 (92.7\%) encounters, respectively.

The predictive validity for presumed sepsis of all scores was poor (SIRS: AUCROC $=0.55[0.54-0.56]$; SOFA: AUCROC $=0.67$ [0.65-0.68]; qSOFA: AUCROC $=0.61[0.60-0.63]$; SIRS vs. SOFA: $p<0.001$; qSOFA vs. SOFA: $p<0.001$; SIRS vs. qSOFA: $p<0.001$; Fig. 2 ). While the SIRS criteria and qSOFA score revealed high grades of sensitivity and low specificity, contrary results were demonstrated for the SOFA score (Table 2).

Moreover, mortality among the encounters with suspected infection on the IMCU/ICU amounted to 163 (9.4\%). All of them offered a positive qSOFA score and at least two positive SIRS criteria (163 [100\%]), while the SOFA score was increased in 119 (73.0\%) encounters.

Regarding hospital mortality, the SIRS criteria and qSOFA score revealed only poor predictive validity, whereas the SOFA score was predictive for the patients' death (SIRS: AUCROC $=0.54$ [0.53-0.54]; SOFA: AUCROC $=0.73$ [CI, 0.70-0.77]; qSOFA: AUCROC = 0.59 [0.58-0.59]; SIRS vs. SOFA: $p<0.001$; qSOFA vs. SOFA: $p<0.001$; SIRS vs. qSOFA: $p<0.001$; Fig. 3). SIRS criteria and qSOFA score reached high sensitivity

Table 2 Sensitivity and specificity of clinical scores for infection

\begin{tabular}{llll}
\hline Parameter & IMCU & ICU & IMCU/ICU \\
\hline Sensitivity of SIRS & 0.78 & 0.93 & 0.97 \\
Specificity of SIRS & 0.48 & 0.32 & 0.13 \\
Sensitivity of SOFA & 0.05 & 0.33 & 0.42 \\
Specificity of SOFA & 0.99 & 0.97 & 0.92 \\
Sensitivity of qSOFA & 0.56 & 0.81 & 0.93 \\
Specificity of qSOFA & 0.70 & 0.51 & 0.30 \\
\hline
\end{tabular}

ICU Intensive care unit, IMCU Intermediate care unit, SIRS Systemic inflammatory response syndrome, SOFA Sequential Organ Failure Assessment, qSOFA Quick Sequential Organ Failure Assessment but low specificity regarding mortality, whereas the SOFA score performed adequately (Table 3 ).

\section{Discussion}

The rapid identification of sepsis serves as the basis for its successful management. According to the current recommendations of the Surviving Sepsis Campaign, the SOFA score should be used as a predictive tool for the detection of sepsis as well as for the risk stratification of critically ill patients. In addition, the qSOFA score has been introduced for the identification of septic patients outside of the ICU $[7,20,21]$. However, to our knowledge, both scores have not been evaluated in the context of surgical IMCU patients. Therefore, this is the first study comparing the predictive value for presumed sepsis of the SOFA and qSOFA scores, as well as the 1992 defined SIRS criteria, in a large cohort of 13,780 surgical IMCU and ICU patients of a tertiary university hospital.

Overall, among encounters with suspected infection in IMCU patients, none of the analyzed scoring tools showed sufficient predictive validity for severe infection (defined as the use of broad-spectrum antibiotics), whereas the qSOFA score was able to predict mortality in a sufficient manner. Interestingly, even though the assessment with the historical SIRS criteria does not meet the current practice guidelines, they were more predictive than the SOFA score within the IMCU patient cohort. Furthermore, among ICU patients as well as patients who underwent a combined IMCU and ICU treatment, all analyzed scoring systems failed to provide sufficient validity for the prediction of infection and mortality. Only in patients who underwent a combined IMCU and ICU treatment the SOFA score was able to adequately predict mortality.

At first glance, these study results might be surprising. However, in comparison to previous findings, the 

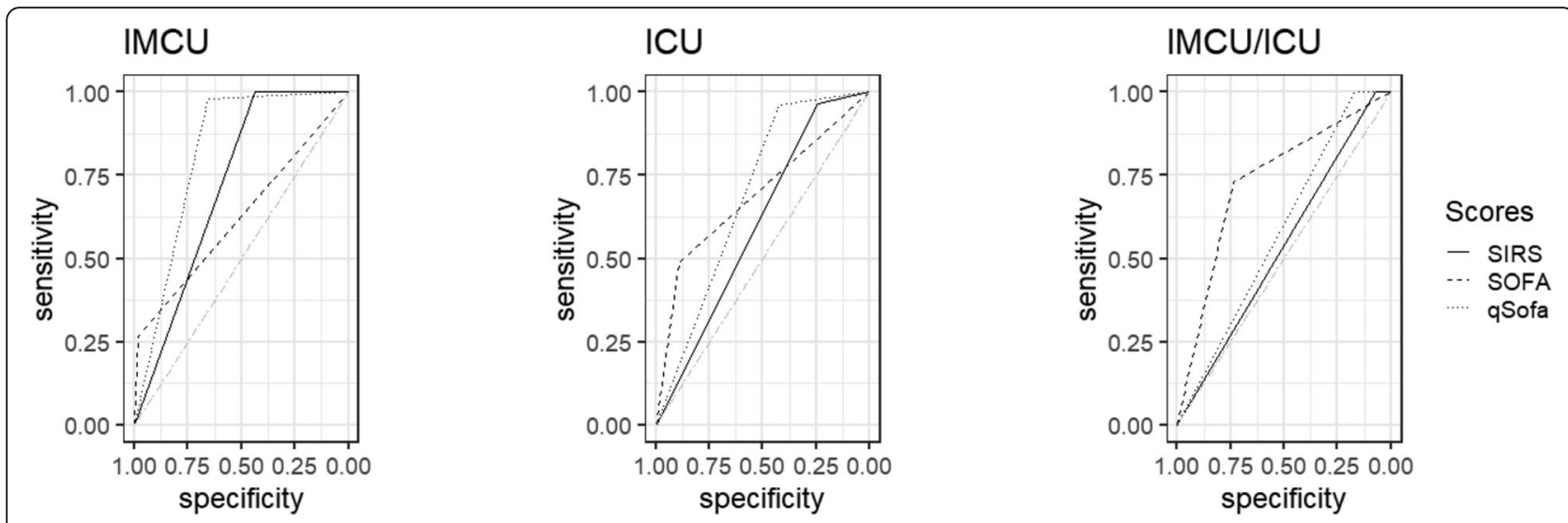

Fig. 3 Predictive validity for hospital mortality using clinical scores. Receiver operating characteristic curves for positive SIRS criteria, SOFA score, and qSOFA score are pictured. ICU, intensive care unit; IMCU, intermediate care unit; qSOFA, quick Sequential Organ Failure Assessment; SIRS, systemic inflammatory response syndrome; SOFA, Sequential Organ Failure Assessment

performance of the qSOFA score and the SIRS criteria remain agreeable. The qSOFA score was first developed and validated by Seymour et al., who analyzed 148,907 unselected patient encounters with suspected infection, consisting of a validation cohort of 7932 ICU and 66,522 non-ICU patients. With the exception of the SOFA score, the predictive value of the qSOFA and SIRS criteria could be matched to our study results within the ICU cohort (AUCROC SOFA 0.74 vs. 0.52; AUCROC qSOFA 0.66 vs. 0.63; AUCROC SIRS 0.64 vs. 0.63) [7]. Their findings have been validated in several studies featuring large numbers of patients (Table 4), resulting in a varying performance of the mentioned scores. However, it has to be stressed that originally Seymour et al. aimed to validate the $\mathrm{qSOFA}$ and SOFA scores as predictors for mortality and not for the identification of sepsis. The Sepsis-3 definition indicates that, due to their predictive value for mortality, both scores can be used for sepsis risk stratification (either at the ICU or non-ICU), but also emphasizes that the underlying data was derived from retrospective studies and requires further validation. However, until now, no prospective data, with sufficient numbers of patients, is available.

Table 3 Sensitivity and specificity of clinical scores for mortality

\begin{tabular}{llll}
\hline Parameter & IMCU & ICU & IMCU/ICU \\
\hline Sensitivity of SIRS & 1.00 & 0.96 & 1.00 \\
Specificity of SIRS & 0.44 & 0.24 & 0.07 \\
Sensitivity of SOFA & 0.27 & 0.49 & 0.73 \\
Specificity of SOFA & 0.98 & 0.89 & 0.74 \\
Sensitivity of qSOFA & 0.98 & 0.96 & 1.00 \\
Specificity of qSOFA & 0.65 & 0.42 & 0.17 \\
\hline
\end{tabular}

ICU Intensive care unit, IMCU Intermediate care unit, SIRS Systemic inflammatory response syndrome, SOFA Sequential Organ Failure Assessment, qSOFA Quick Sequential Organ Failure Assessment
Lo et al. performed a literature review and retrospective data analysis of 380,920 patients, demonstrating an AUCROC of 0.68 for the predictive value of in-hospital mortality for the qSOFA score, which is in line with our study findings in surgical ICU and IMCU patients [20]. Furthermore, a meta-analysis of 229,480 patients compared the qSOFA score and SIRS criteria for their ability to predict patient mortality and revealed only a slightly better performance of the qSOFA score, which supports the findings of our study [22]. However, some studies revealed a high power for the prediction of mortality: Kovach et al. analyzed hospital mortality in a retrospective data set of 3749 surgical and medical ICU patients with suspected infection, while Zhang et al. investigated retrospectively 5109 cardiac surgical patients, with both studies resulting in AUCROC $>0.8$ for the prediction of mortality by using the SOFA and qSOFA scores [21, 23]. However, it must be highlighted that, contrary to our approach, the patients of Kovach's study were adjusted for a baseline risk factor for death, which increased the predictive quality of the SOFA score, while Zhang et al. only included cardiac surgical patients, which are hardly comparable with the sources of systemic inflammation in our study. During cardiac surgery, systemic inflammation is mainly induced by cardiopulmonary bypass, which leads to strong activation of the inflammatory response through the blood's foreign surface contact with the components of the heart-lung machine, reperfusion injury/reperfusion injury [24]. Contrarily, local surgical trauma is causative for the onset of inflammation during non-cardiac surgery.

Even though qSOFA and SOFA scores are widely accepted as tools for the identification of septic patients, they failed to predict suspected infection in each individual group of patients in our study. These findings are supported by Krebs et al., who also evaluated the qSOFA and 
Table 4 Overview studies regarding clinical criteria

\begin{tabular}{|c|c|c|c|c|c|c|c|}
\hline Author & Patients & Collective & Primary outcome & $\begin{array}{l}\text { Suspected } \\
\text { infection }\end{array}$ & SIRS & SOFA & qSOFA \\
\hline Lo et al. [20] & $n=380,920$ & Mixed & Mortality & No & n.a. & n.a. & 0.68 \\
\hline Kovach et al. [21] & $n=10,981$ & ED; ICU; mixed & Mortality & Yes & 0.79 & 0.90 & 0.84 \\
\hline Seymour et al. [7] & $n=7932$ & ICU; mixed & Mortality & Yes & 0.64 & 0.74 & 0.66 \\
\hline Zhang et al. [23] & $n=5109$ & Surgical ICU & Mortality & No & 0.95 & 0.96 & 0.95 \\
\hline Falcao et al. [31] & $n=3008$ & Surgical ICU & Mortality & No & n.a. & 0.742 & n.a. \\
\hline Gando et al. [25] & $n=1045$ & ED; mixed & Infection & Yes & 0.647 & n.a. & 0.582 \\
\hline Basile-Filho et al. [32] & $n=847$ & Surgical ICU & Mortality & No & n.a. & 0.791 & n.a. \\
\hline Mungan et al. [33] & $n=233$ & Surgical ICU & Mortality & No & n.a. & 0.631 & n.a. \\
\hline Innocenti et al. [34] & $n=135$ & ED-HDU; mixed & Mortality & Yes & n.a. & 0.80 & n.a. \\
\hline
\end{tabular}

ICU Intensive care unit, ED Emergency department, HDU High-dependency unit, qSOFA Quick Sequential Organ Failure Assessment, SIRS Systemic inflammatory response syndrome, SOFA Sequential organ failure assessment

SOFA scores as well as the SIRS criteria in 1942 prospective patient days within a cohort of surgical trauma ICU patients, concluding that all scores failed to predict the development of new infections [17]. But, also in an out-of-ICU setting, a failure of the qSOFA score (and SIRS criteria) has already been described in a collective of patients attending the emergency room $(n=1045)$ [25]. Moreover, another large retrospective analysis failed to prove a high predictive power of the qSOFA score and the SIRS criteria in patients admitted to the emergency department [26].

These opposing results might be partially explainable, as already discussed above, by the choice of the study population, which might strongly influence the study results because only postsurgical patients were investigated in our study, in contrast to medical and surgical patients in the underlying study. Further, the variable predictive validity between the studies might be caused by the differences in the study designs. Considering the original publication of Seymour et al., the lower predictive capacity of the SOFA score in our study might be caused by the varying definition of suspected infection. While it was defined as the combination of antibiotics and body fluid cultures by Seymour et al., the administration of broad-spectrum antibiotics was used in our approach. It has to be noted that the prescribing behavior of antibiotics varies between physicians depending on their clinical experience, qualification, and specialty. Charani et al. compared the antibiotic prescribing between medical and surgical specialties. Besides more frequent and longer prescription, antibiotics were more likely to be escalated in surgical patients [27]. A recent systemic review offers a potential explanation for these findings by identifying nine determinants that influenced antibiotic prescription behavior including the fear of risking an adverse outcome [28]. Surgical patients are challenging when it comes to identifying infectious complications, and the consequences of sepsis are more devastating in these patients which potentially leads to a more liberal application of broad-spectrum antibiotics [29, 30]. This might offer an explanation for the low specificity of the analyzed scores for detecting a presumed sepsis. Furthermore, even in an isolated analysis of studies that only investigated surgical patients, the predictive performance varies strongly: Falcao et al. analyzed 3008 surgical ICU patients and showed a sufficient predictive validity of the SOFA score regarding mortality (AUCROC of 0.74) [31]. Similar results are published by Basile-Filho et al., who revealed an AUCROC of 0.79 by using the SOFA score for the prediction of mortality within 847 surgical ICU patients [32]. Contrarily, Mungan et al. showed a lower predictive validity of 0.63 of the SOFA score in surgical ICU patients [33]. Although authors of these studies described their population as "surgical patients," it must be highlighted that their calculations comprised all kinds of surgical patients, independently of their risk for infection, including those without suspicion of infection. By contrast, our study only focused on the investigation of postsurgical patients with suspected infection. In our opinion, this issue is of high relevance because the majority of postsurgical patients following major surgery regularly show clinical signs of systemic inflammation such as tachycardia, fever, and tachypnea, which commonly represent signs of a surgery-induced systemic inflammation rather than an infection. For this reason, it is not only challenging to discriminate between postsurgical sterile systemic inflammation and infection, but the predictive ICU sores might also become distorted into false positive results. This may explain the high sensitivity but low specificity of the qSOFA score and SIRS criteria because both systems include only clinical criteria for easy assessment. Since these criteria are often fulfilled during the postsurgical phase, the chance that they are truly positive is high (sensitivity). On the other hand, this leads to a low rate of true false cases (specificity). Since the SOFA score consists of much more detailed intensive care variables than the qSOFA score and the 
SIRS criteria, the specificity is higher, but sensitivity remains low. These arguments are in accordance with the findings of Gando et al. as well as Krebs et al., who demonstrated that the SIRS criteria, SOFA score, and qSOFA score were not able to predict sepsis in the emergency department or surgical ICU $[17,25]$.

These limitations of the ICU scores are of high interest for their use on surgical IMCUs because of the increasing demand of IMCU capacity. Therefore, the importance of the surgical IMCU, as a bridge to the normal ward, is rising. Patients attending the IMCU commonly represent those surgical patients at moderate to high risk of developing postsurgical complications. Analogous to ICU patients, the rapid identification of infectious complications is altered by surgery-induced signs of systemic inflammation, underlining the need for specific IMCU scores. Lacking studies that focus on surgical IMCUs, other high-dependency units (HDUs) (but not ICUs) have to be analyzed for the interpretation of our study results. Innocenti et al. analyzed 3311 patients admitted to HDUs and demonstrated that the SOFA score, in opposition to our results, showed a good discriminatory ability for HDU mortality [34]. However, contrary to our approach, no cutoff values for SOFA scores were used, and no postsurgical patients were included. In our study, the prediction of mortality was sufficient using the qSOFA score in IMCU patients. Another study showed that these scores are also not able to predict infection in the emergency room [25]. Based on the findings of our study, the use of the qSOFA score as a predictor of mortality can be supported, while its predictive power for the detection of suspected infection can be doubted in postsurgical IMCU patients, which might be caused by surgery-induced systemic inflammation.

However, due to the retrospective study design, further prospective studies that include high numbers of postsurgical IMCU patients are necessary to validate these findings. Due to the fact that most critically ill patients are regularly transferred to the IMCU during their medical treatment, a subgroup of these patients was created. The increased APACHE II score reflects the serious illness of the included patients. Since clinical scores were not able to distinguish for suspected infection in this subgroup, severity of disease seems not to improve the quality of these scores. While adequate prediction for mortality was calculated with the SOFA score, this could indicate its better quality in critically ill patients. Since these patients are missing in the ICU subgroup, this could also explain our lower results for the SOFA score in the ICU.

Nevertheless, our study features some limitations. First, this retrospective analysis is not able to draw conclusions regarding the underlying causalities. Second, due to the retrospective design, no sample size calculation was performed. Third, lacking of more specific alternatives, the administration of broad-spectrum antibiotic agents was used as a surrogate for the diagnosis of suspected infection. While the clinical symptoms and inflammatory parameters are physiologically altered by the surgery, body fluid cultures result in negative samples in a majority of cases (e.g., due to the perioperative antibiotic treatment) $[28,35,36]$. Furthermore, even if sepsis was assessed by intensivists, its diagnosis remains subjective [37]. Nonetheless, it has to be highlighted that the administration of a broad-spectrum antibiotic agent serves only as a surrogate for the true presence of sepsis. Fourth, the RAAS was used as a surrogate parameter for GCS $<15$ in the absence of the GCS, which is problematical since the qSOFA score was developed and validated with the use of GCS.

\section{Conclusions}

In summary, neither SOFA nor qSOFA score was able to distinguish for suspected sepsis (defined by the application of broad-spectrum antibiotics) in surgical patients, independently of IMCU, ICU, or IMCU/ICU stay. Nevertheless, the qSOFA score revealed sufficient prediction for mortality in the IMCU. Further, as the SOFA score showed the best results regarding mortality in IMCU/ICU patients, its predictive quality depended on the severity of the disease. Summarizing, it remains unclear whether qSOFA or SOFA score should be used in surgical IMCU patients for risk stratification.

\section{Abbreviations}

APACHE II: Acute physiology and chronic health evaluation II; AUROC: Area under the receiver operating curve; GCS: Glasgow coma scale; HDU: Highdependency unit; ICU: Intensive care unit; IQR: Interquartile range; IMCU: Intermediate care unit; qSOFA: Quick sequential organ failure assessment; RAAS: Richmond agitation sedation scale; RR: Respiratory rate; SBP: Systolic blood pressure; SD: Standard deviation; SIRS: Systemic inflammatory response syndrome; SOFA: Sequential organ failure assessment; STROBE: Strengthening the reporting of observational studies in epidemiology

\section{Acknowledgements}

Not applicable

\section{Authors' contributions \\ $\mathrm{CK}, \mathrm{FE}, \mathrm{ES}$, and MS were responsible for the study design and conduct, CK, $F B$, and TF data acquisition, statistical analysis and interpretation, and writing of the manuscript. MM performed the statistical analysis and $\mathrm{CK}, \mathrm{FE}, \mathrm{ES}, \mathrm{AH}$, and CK interpretation, and approved and helped to draft the manuscript. All authors approved the final version of the manuscript.}

\section{Funding}

This study was funded by the German Center for Infection Research. Open Access funding enabled and organized by Projekt DEAL.

\section{Availability of data and materials}

All data generated or analyzed during this study are included in this published article (and its supplementary information files).

\section{Ethics approval and consent to participate}

This study was approved by the local ethics committee (Justus-LiebigUniversity, Giessen, Germany, trial code 240/16). The study methods and results are presented in accordance with STROBE guidelines. 


\section{Consent for publication}

Not applicable.

\section{Competing interests}

The authors declare no competing interests.

\section{Author details}

'Department of Anesthesiology, Operative Intensive Care Medicine and Pain Therapy, University Hospital of Giessen, Rudolf-Buchheim-Street 7, 35392 Giessen, Germany. ${ }^{2}$ German Center of Infection Research (DZIF), Partner Site Giessen/Marburg/Langen, Giessen, Germany. ${ }^{3}$ Department of General and Thoracic Surgery, University Hospital of Giessen, Giessen, Germany.

Received: 4 September 2020 Accepted: 5 November 2020

\section{Published online: 25 November 2020}

\section{References}

1. Singer M, Deutschman CS, Seymour CW, Shankar-Hari M, Annane D, Bauer M, et al. The Third International Consensus Definitions for Sepsis and Septic Shock (Sepsis-3). JAMA. 2016;315:801-10. https://doi.org/10. 1001/jama.2016.0287.

2. Pruinelli L, Westra BL, Yadav P, Hoff A, Steinbach M, Kumar V, et al. Delay within the 3-hour surviving sepsis campaign guideline on mortality for patients with severe sepsis and septic shock. Crit Care Med. 2018;46:500-5. https://doi.org/10.1097/CCM.0000000000002949.

3. Sprung CL, Sakr Y, Vincent J-L, Le Gall J-R, Reinhart K, Ranieri VM, et al. An evaluation of systemic inflammatory response syndrome signs in the Sepsis Occurrence in Acutely ill Patients (SOAP) study. Intensive Care Med. 2006;32: 421-7. https://doi.org/10.1007/s00134-005-0039-8.

4. Dulhunty JM, Lipman J, Finfer S. Does severe non-infectious SIRS differ from severe sepsis? Intensive Care Med. 2008;34:1654-61. https://doi.org/10.1007/ s00134-008-1160-2

5. Levy MM, Fink MP, Marshall JC, Abraham E, Angus D, Cook D, et al. 2001 SCCM/ESICM/ACCP/ATS/SIS International Sepsis Definitions Conference. Crit Care Med. 2003;31:1250-6. https://doi.org/10.1097/01.CCM.0000050454. 01978.3B.

6. Vincent JL, Moreno R, Takala J, Willatts S, de Mendonça A, Bruining H, et al. The SOFA (Sepsis-related organ failure assessment) score to describe organ dysfunction/failure. On behalf of the working group on sepsis-related problems of the European society of intensive care medicine. Intensive Care Med. 1996:22:707-10. https://doi.org/10.1007/BF01709751.

7. Seymour CW, Liu VX, Iwashyna TJ, Brunkhorst FM, Rea TD, Scherag A, et al. Assessment of clinical criteria for sepsis: for the Third International Consensus Definitions for Sepsis and Septic Shock (Sepsis-3). JAMA. 2016; 315:762-74. https://doi.org/10.1001/jama.2016.0288.

8. Wendlandt B, Bice T, Carson S, Chang L. Intermediate care units: a survey of organization practices across the United States. J Intensive Care Med. 2020; 35:468-71. https://doi.org/10.1177/0885066618758627.

9. Halpern NA, Pastores SM. Critical care medicine in the United States 20002005: an analysis of bed numbers, occupancy rates, payer mix, and costs*. Crit Care Med. 2010;38:65-71. https://doi.org/10.1097/CCM. 0b013e3181b090d0.

10. Waydhas C, Herting E, Kluge S, Markewitz A, Marx G, Muhl E, et al. Intermediate care station : Empfehlungen zur Ausstattung und Struktur. Med Klin Intensivmed Notfmed. 2018;113:33-44. https://doi.org/10.1007/ s00063-017-0369-7.

11. Plate JDJ, Leenen LPH, Houwert M, Hietbrink F. Utilisation of intermediate care units: a systematic review. Crit Care Res Pract. 2017:2017:8038460. https://doi.org/10.1155/2017/8038460.

12. Plate JDJ, Peelen $L M$, Leenen $L P H$, Houwert RM, Hietbrink F. Assessment of the intermediate care unit triage system. Trauma Surg Acute Care Open. 2018:3:e000178. https://doi.org/10.1136/tsaco-2018-000178.

13. Hamsen U, Lefering R, Fisahn C, Schildhauer TA, Waydhas C. Workload and severity of illness of patients on intensive care units with available intermediate care units: a multicenter cohort study. Minerva Anestesiol. 2018;84:938-45. https://doi.org/10.23736/50375-9393.18.12516-8.

14. Capuzzo M, Volta C, Tassinati T, Moreno R, Valentin A, Guidet B, et al. Hospital mortality of adults admitted to intensive care units in hospitals with and without intermediate care units: a multicentre European cohort study. Crit Care. 2014;18:551. https://doi.org/10.1186/s13054-014-0551-8,
15. Meaudre E, Nguyen C, Contargyris C, Montcriol A, d'Aranda E, Esnault $P$, et al. Management of septic shock in intermediate care unit. Anaesth Crit Care Pain Med. 2018;37:121-7. https://doi.org/10.1016/j.accpm.2017.07.004.

16. Vincent J-L. The clinical challenge of sepsis identification and monitoring. PLoS Med. 2016;13:e1002022. https://doi.org/10.1371/journal.pmed.1002022.

17. Krebs ED, Hassinger TE, Guidry CA, Berry PS, Elwood NR, Sawyer RG. Non-utility of sepsis scores for identifying infection in surgical intensive care unit patients. Am J Surg. 2019;218:243-7. https://doi.org/10.1016/j.amjsurg.2018.11.044.

18. Rhodes A, Evans LE, Alhazzani W, Levy MM, Antonelli M, Ferrer R, et al. Surviving Sepsis Campaign: international guidelines for management of sepsis and septic shock: 2016. Intensive Care Med. 2017;43:304-77. https:// doi.org/10.1007/s00134-017-4683-6.

19. Bone RC, Balk RA, Cerra FB, Dellinger RP, Fein AM, Knaus WA, et al. Definitions for sepsis and organ failure and guidelines for the use of innovative therapies in sepsis. The ACCP/SCCM Consensus Conference Committee. American College of Chest Physicians/Society of Critical Care Medicine. Chest. 1992;101:1644-55. https://doi.org/10.1378/chest.101.6.1644.

20. Lo RSL, Leung LY, Brabrand M, Yeung CY, Chan SY, Lam CCY, et al. qSOFA is a poor predictor of short-term mortality in all patients: a systematic review of 410,000 patients. J Clin Med. 2019. https://doi.org/10.3390/jcm8010061.

21. Kovach CP, Fletcher GS, Rudd KE, Grant RM, Carlbom DJ. Comparative prognostic accuracy of sepsis scores for hospital mortality in adults with suspected infection in non-ICU and ICU at an academic public hospital. PLoS One. 2019;14:e0222563. https://doi.org/10.1371/journal.pone.0222563.

22. Serafim R, Gomes JA, Salluh J, Póvoa P. A comparison of the quick-SOFA and systemic inflammatory response syndrome criteria for the diagnosis of sepsis and prediction of mortality: a systematic review and meta-analysis. Chest. 2018;153:646-55. https://doi.org/10.1016/j.chest.2017.12.015.

23. Zhang $Y$, Luo $H$, Wang $H$, Zheng $Z$, Ooi OC. Validation of prognostic accuracy of the SOFA score, SIRS criteria, and GSOFA score for in-hospital mortality among cardiac-, thoracic-, and vascular-surgery patients admitted to a cardiothoracic intensive care unit. J Card Surg. 2020;35:118-27. https:// doi.org/10.1111/jocs.14331.

24. Millar JE, Fanning JP, McDonald Cl, McAuley DF, Fraser JF. The inflammatory response to extracorporeal membrane oxygenation (ECMO): a review of the pathophysiology. Crit Care. 2016. https://doi.org/10.1186/s13054-016-1570-4.

25. Gando S, Shiraishi A, Abe T, Kushimoto S, Mayumi T, Fujishima S, et al. The SIRS criteria have better performance for predicting infection than qSOFA scores in the emergency department. Sci Rep. 2020;10:8095. https://doi.org/ 10.1038/s41598-020-64314-8

26. Tusgul S, Carron P-N, Yersin B, Calandra T, Dami F. Low sensitivity of qSOFA, SIRS criteria and sepsis definition to identify infected patients at risk of complication in the prehospital setting and at the emergency department triage. Scand J Trauma Resusc Emerg Med. 2017;25:108. https://doi.org/10. 1186/s13049-017-0449-y.

27. Charani E, de Barra E, Rawson TM, Gill D, Gilchrist M, Naylor NR, Holmes AH. Antibioctic prescribing in general medical and surgical specialties: a prospective cohort study. Antimicrob Resist Infect Control. 2019;8:151. https://doi.org/10.1186/s13756-019-0603-6.

28. Warreman EB, Lambregts MMC, Wouters RHP, Visser LG, Staats $H$, van Dijk E, de Boer MGJ. Determinants of in-hospital antibiotic prescription behaviour: a systematic review and formation of a comprehensive framework. Clin Microbiol Infect. 2019:25:538-45.

29. Shapiro N, Howell MD, Bates DW, Angus DC, Ngo L, Talmor D. The association of sepsis syndrome and organ dysfunction with mortality in emergency department patients with suspected infection. Ann Emerg Med. 2006;48:583-90, 590.e1. https://doi.org/10.1016/j.annemergmed.2006.07.007.

30. Cheng B, Xie G, Yao S, Wu X, Guo Q, Gu M, et al. Epidemiology of severe sepsis in critically ill surgical patients in ten university hospitals in China. Crit Care Med. 2007;35:2538-46. https://doi.org/10.1097/01.CCM.0000284492. 30800.00 .

31. Falcão ALE, AGdA B, AAM B, Ferreira NL, Logato CM, Silva FP, et al. The prognostic accuracy evaluation of SAPS 3, SOFA and APACHE II scores for mortality prediction in the surgical ICU: an external validation study and decision-making analysis. Ann Intensive Care. 2019;9:18. https://doi.org/10. 1186/s13613-019-0488-9.

32. Basile-Filho A, Lago AF, Menegueti MG, Nicolini EA, LAdB R, Nunes RS, et al. The use of APACHE II, SOFA, SAPS 3, C-reactive protein/albumin ratio, and lactate to predict mortality of surgical critically ill patients: a retrospective cohort study. Medicine (Baltimore). 2019;98:e16204. https://doi.org/10.1097/ MD.0000000000016204. 
33. Mungan I, Bektaş Ş, Alıınkaya Çavuş M, Sarı S, Turan S. The predictive power of SAPS-3 and SOFA scores and their relations with patient outcomes in the surgical intensive care unit. Turk J Surg. 2019;35:124-30. https://doi.org/10. 5578/turkjsurg.4223.

34. Innocenti F, Bianchi S, Guerrini E, Vicidomini S, Conti A, Zanobetti M, Pini R. Prognostic scores for early stratification of septic patients admitted to an emergency department-high dependency unit. Eur J Emerg Med. 2014;21: 254-9. https://doi.org/10.1097/MEJ.0000000000000075.

35. Lam SW, Bauer SR, Fowler R, Duggal A. Systematic review and meta-analysis of procalcitonin-guidance versus usual care for antimicrobial management in critically ill patients: focus on subgroups based on antibiotic initiation, Cessation, or Mixed Strategies. Crit Care Med. 2018;46:684-90. https://doi. org/10.1097/CCM.0000000000002953.

36. Schuetz P, Wirz Y, Sager R, Christ-Crain M, Stolz D, Tamm M, et al. Effect of procalcitonin-guided antibiotic treatment on mortality in acute respiratory infections: a patient level meta-analysis. Lancet Infect Dis. 2018;18:95-107. https://doi.org/10.1016/S1473-3099(17)30592-3.

37. Rhee C, Kadri SS, Danner RL, Suffredini AF, Massaro AF, Kitch BT, et al. Diagnosing sepsis is subjective and highly variable: a survey of intensivists using case vignettes. Crit Care. 2016;20:89. https://doi.org/10.1186/s13054016-1266-9.

\section{Publisher's Note}

Springer Nature remains neutral with regard to jurisdictional claims in published maps and institutional affiliations.

Ready to submit your research? Choose BMC and benefit from:

- fast, convenient online submission

- thorough peer review by experienced researchers in your field

- rapid publication on acceptance

- support for research data, including large and complex data types

- gold Open Access which fosters wider collaboration and increased citations

- maximum visibility for your research: over $100 \mathrm{M}$ website views per year

At BMC, research is always in progress.

Learn more biomedcentral.com/submissions 